\title{
Cloning and characterization of a novel 2-ketoisovalerate reductase from the beauvericin producer Fusarium proliferatum LF061
}

\author{
Tao Zhang ${ }^{1,2}$, XiaoPeng Jia ${ }^{1,2}$, Ying Zhuo ${ }^{1}$, Mei Liu ${ }^{1}$, Hong Gao ${ }^{1}$, JinTao Liu ${ }^{1,2^{\wedge}}$ and Lixin Zhang ${ }^{1 *}$
}

\begin{abstract}
Background: The ketoisovalerate reductase (EC 1.2.7.7) is required for the formation of beauvericin via the nonribosomal peptide synthetase biosynthetic pathway. It catalyzes the NADPH-specific reduction of ketoisovaleric acid to hydroxyisovalerate. However, little is known about the bioinformatics' data about the 2-Kiv reductase in Fusarium. To date, heterologous production of the gene KivRFp from Fusarium has not been achieved.

Results: The KivRFp gene was subcloned and expressed in Escherichia coli BL21 using the pET expression system. The gene KivRFp contained a 1,359 bp open reading frame (ORF) encoding a polypeptide of 452 amino acids with a molecular mass of $52 \mathrm{kDa}$. Sequence analysis indicated that it showed $61 \%$ and $52 \%$ amino acid identities to ketoisovalerate reductase from Beauveria bassiana ATCC 7159 (ACl30654) and Metarhizium acridum CQMa 102 (EFY89891), respectively; and several conserved regions were identified, including the putative nucleotide-binding signature site, GXGXXG, a catalytic triad (Glu405, Asn184, and Lys285). The KivRFp exhibited the highest activity at $35^{\circ} \mathrm{C}$ and $\mathrm{pH} 7.5$ respectively, by reduction of ketoisovalerate. It also exhibited the high level of stability over wide temperature and $\mathrm{pH}$ spectra and in the presence of metal ions or detergents.
\end{abstract}

Conclusions: A new ketoisovalerate reductase KivRFp was identified and characterized from the depsipeptide-producing fungus F. proliferatum. KivRFp has been shown to have useful properties, such as moderate thermal stability and broad pH optima, and may serve as the starting points for future protein engineering and directed evolution, towards the goal of developing efficient enzyme for downstream biotechnological applications.

Keywords: 2-Kiv Reductase, Beauvericin, Fusarium proliferatum LF061

\section{Background}

Cyclooligomer depsipeptides (CODs) are a prominent class of bioactive peptides produced from various Fungi by large multimeric enzyme complexes called nonribosomal peptide synthetases (NRPSs). There are many reports about CODs which contain various hydroxyl acids, such as D-hydroxyisovalerate (D-Hiv); examples include the beauvericin [1], enniatins [2], destruxin [3], bassianolide [4], fusafungine [5]. Beauvericin, produced by certain fungi of Beauveria [1], Isaria [6] and Fusarium [7] that exhibits insecticidal, displays antimicrobial,

\footnotetext{
* Correspondence: zhanglixin@im.ac.cn

'Deceased

${ }^{1}$ Chinese Academy of Sciences Key Laboratory of Pathogenic Microbiology and Immunology, Institute of Microbiology, Chinese Academy of Sciences, Bei'er Tiao Road, Zhongguancun Haidian District, Beijing 100190, China Full list of author information is available at the end of the article
}

anti-tumor activities, reverses multidrug resistance in Candida albicans [8-11]. Its molecular structure consists of an alternating sequence of three $\mathrm{N}$-methyl-L-phenylalanine and three D-hydroxyisovaleric acids (Figure 1) [12]. Thus, the hydroxyl acid is a key intermediate in the formation of the beauvericin.

In mammals, the catabolism of the branched-chain amino acids (BCAAs) leucine, isoleucine, and valine proceeds by two-step process, i.e. transamination step (aminotransfer) and oxidation step. BCAAs are usually transaminated to form 2-keto acids by branched-chain amino acid transaminases (BCATs). These 2-keto acids are oxidatively decarboxylated by mitochondrial branchedchain 2-ketoacid dehydrogenase [13-15] and converted to acyl CoA. However, in some fungal species, namely the hydroxysiovalerate-containing CODs producers, there is another pathway leading from L-Val to D-Hiv via the keto

\section{Biomed Central}




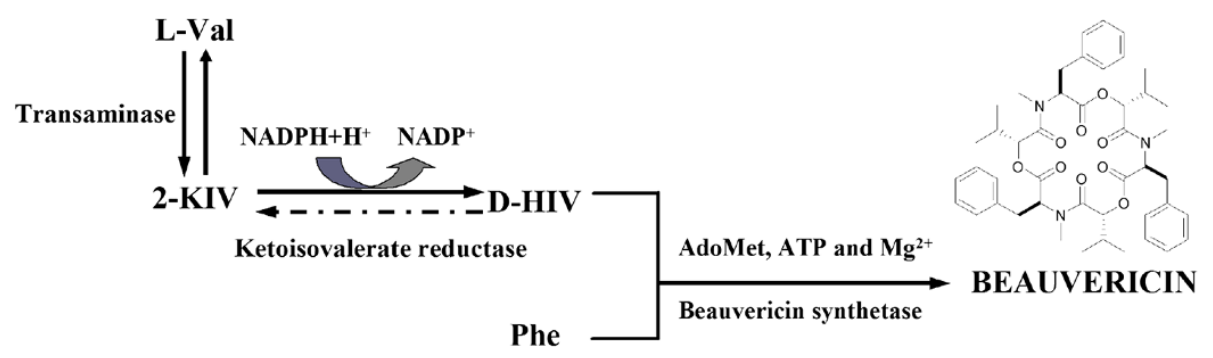

Figure 1 Overall mechanism of beauvericin biosynthesis in Fusarium species.

acid (Figure 1). D-hydroxyisovalerate dehydrogenase, catalyzes the reversible reduction of 2-ketoisovalertate (2-Kiv), the intermediate of branched-chain amino acid metabolism [16], to D-Hiv in the presence of NADPH, which cannot be replaced by NADH [17-19]. So far, D-HivDH has been purified and partially characterized exclusively from the enniatin producer, Fusarium sambucinum $[17,19]$, but the corresponding protein and gene sequences were not determined. $\mathrm{Xu}$ et al., demonstrated the novel kivr-encoding 2-ketoisovalerate reductase is the sole supplier of D-Hiv for both beauvericin and bassianolide biosynthesis in Beauveria bassiana ATCC 7159 [20].

On the other hand, little is known about the bioinformatics' data about the 2-Kiv reductase (2-KivR) in Fusarium, thus warranting further study. To date, heterologous production of the gene KivRFp from Fusarium has not been achieved; similarly, very little information is available about the role of the KivRFp in the regulation of beauvericin biosynthesis. In order to get a closer insight into the unique and intriguing features of the ketoisovalerate reductase in the Fusarium genus, we undertook both genetic and biochemical studies. In the present article, we describe the identification and functional analysis of the KivRFp of Fusarium proliferatum LF061. Our report demonstrates that the KivRFp constituted a new member of family ketonate oxidoreductases.

\section{Methods}

\section{Chemicals}

Chemicals were of the highest purity commercially available. Pyruvate, 2-Kiv, 2-ketovalerate, 2-ketoglutarate and 2-ketobutyrate were obtained from TCI Japan Ltd. D-Hiv were purchased from Sigma. NADH, NADPH, NADP ${ }^{+}$ were products of Roche.

\section{Bacterial strains, plasmids, and culture}

The starting strains and plasmids used in this study are listed in Table 1. E. coli was grown at $37^{\circ} \mathrm{C}$ in Luria-Bertani (LB) medium supplemented with appropriate antibiotics [21]. When required, ampicillin was added at a final concentration of $100 \mu \mathrm{g} / \mathrm{mL}$, kanamycin at $25 \mu \mathrm{g} / \mathrm{mL}$, and chloramphenicol, at $12.5 \mu \mathrm{g} / \mathrm{mL}$. Fusarium proliferatum LF061 (deposited in China General Microbiological
Culture Collection under the accession number of CGMCC 3.1777) was maintained on potato dextrose agar (PDA).

\section{DNA preparation and manipulation}

E. coli cells were transformed by the calcium chloride procedure [21]. Recombinant plasmid DNA was isolated by the method of Birnboim and Doly [22]. For sequencing, this DNA was further purified by polyethylene glycol precipitation [21]. Restriction enzymes, T4 DNA ligase and calf intestinal alkaline phosphatase were purchased from New England Biolabs (Ipswich, USA) or Takara (Tokyo, Japan) and used according to the manufacturers' instructions. BugBuster Ni-NTA His. Bind Purification Kit was purchased from Novagen (Code No. NV70751-3, Novagen).

Table 1 Starting bacterial strains and plasmids used in this study

\begin{tabular}{|c|c|c|}
\hline Strain or plasmid & Description & Source \\
\hline \multicolumn{3}{|l|}{ Strains } \\
\hline E. coli TOP10 & 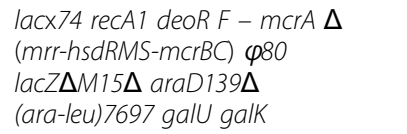 & Transgen \\
\hline E. coli BL21(DE3) & $\begin{array}{l}F-, \text { ompT, hsdSB (rB-, mB-), } \\
d c m, g a l, \lambda(D E 3), p L y s S, C m r\end{array}$ & Novagen \\
\hline $\begin{array}{l}\text { Fusarium proliferatum } \\
\text { LF061 }\end{array}$ & $\begin{array}{l}\text { Wild strain, producing beauvericin } \\
\text { isolated from marine sample }\end{array}$ & This study \\
\hline E. coli TOP10-KivRFp & $\begin{array}{l}\text { Positive clone of artificial gene } \\
\text { synthesis, which carries the } \\
\text { KivRFp gene fragment }\end{array}$ & This study \\
\hline E. coli BL21(DE3)-KivRFp & $\begin{array}{l}\text { Positive clone, which carries the } \\
\text { pKivRFp-His expression vector }\end{array}$ & This study \\
\hline \multicolumn{3}{|l|}{ Plasmids } \\
\hline pUCE & Cloning vector; $A p^{r}$ & Inovogen \\
\hline pET28a & Expression vector; $\mathrm{Km}^{\mathrm{r}}$ & Novagen \\
\hline pUCE-KivRFp & $\begin{array}{l}\text { pUCE, which carries the complete } \\
\text { ketoisovalerate reductase (KivRFp) }\end{array}$ & This study \\
\hline $\mathrm{pKivRFp-His}$ & $\begin{array}{l}\text { pET28a carrying amplified } \\
\text { EcoRI-Ndel fragment containing } \\
\text { ketoisovalerate reductase gene } \\
\text { (KivRFp) }\end{array}$ & This study \\
\hline
\end{tabular}

$\mathrm{Ap}^{\mathrm{r}}$, ampicillin resistant; $\mathrm{Km}^{\mathrm{r}}$, kanamycin resistant. 


\section{Phylogenetic analysis}

Deduced amino acid sequences of 13 ketonate reductases or homologous proteins were subjected to protein phylogenetic analysis. A phylogenetic tree was generated using the neighbor joining method of Saitou and Nei [23] with MEGA 4.0 software [24]. A total of 4 sequences were aligned with the CLUSTAL_W program [25] and visually examined with BoxShade Server program. The length of each branch pair represents the evolutionary distance between the sequences.

\section{Heterologous expression of gene KivRFp and purification of recombinant KivRFp}

Artificial gene synthesis was finished by Innovogen Tech. Co. (Beijing, China). Jcat (Java Codon Adaptation Tool) [26] and OPTIMIZER [27] were used to improve heterologous protein production. The integrity of the nucleotide sequence of all newly constructed plasmids was confirmed by DNA sequencing. The primer pairs with restriction enzyme sites for HindIII and NdeI were designed to generate an $\mathrm{N}$-terminal His-tag of the recombinant ketonate reductase. The modified KivRFp gene was cloned into an expression vector, pET28a (+) and the recombinant plasmid $\mathrm{pKivRFp-His} \mathrm{was} \mathrm{trans-}$ formed into E. coli BL21 (DE3) cells. When the cell density at $600 \mathrm{~nm}$ reached around 0.6, expression of recombinant KivRFp protein was initiated by addition of $0.6 \mathrm{mM}$ isopropylthio- $\beta$-D-galactoside and continued cultivation for additional $6 \mathrm{~h}$ at $20^{\circ} \mathrm{C}$. Cells were harvested by centrifugation at $6,000 \times \mathrm{g}$ for $5 \mathrm{~min}$, washed twice with ice-cold $50 \mathrm{mM}$ sodium phosphate buffer (pH 8.0) and resuspended in the same buffer containing $10 \mathrm{mM}$ imidazole, disrupted by sonification in an icewater bath (60 times, 5 s). Recombinant KivRFp reductase was applied to metal-chelating chromatography using Ni-NTA affinity chromatography (Novagen) according to the manufacturer's instructions.

Polyacrylamide gel electrophoresis of enzyme in the presence of sodium dodecyl sulfate (SDS) was carried out by the method of Sambrook and Russell [21]. Protein concentrations were determined by a modified Bradford procedure with bovine serum albumin as a standard [28].

\section{Enzyme assays}

The standard 2-Kiv reductase assay mixture contained $50 \mathrm{mM}$ sodium phosphate buffer ( $\mathrm{pH}$ 7.0), $0.7 \mathrm{mM}$ 2-Kiv, $0.29 \mathrm{mM} \mathrm{NADPH}$, and enzyme in a final volume of $3.0 \mathrm{~mL}$. The reaction was initiated by the addition of substrate, and the decrease in absorbance at $340 \mathrm{~nm}\left(\mathrm{~A}_{340}\right)$ was measured at $35^{\circ} \mathrm{C}$, which was performed using an UNICO 2802 UV/VIS spectrophotometer. A molar extinction coefficient of $6.22 \mathrm{~cm}^{2} / \mathrm{pmol} \mathrm{NADPH}$ was used for the calculation of enzyme activity. One unit was defined as the amount of enzyme caused the oxidation of $1 \mu \mathrm{mol}$ of NADPH per min. The reaction mixture for the assay of the reverse reaction contained $50 \mathrm{mM}$ sodium phosphate buffer ( $\mathrm{pH}$ 8.0), $2.8 \mathrm{mM} \mathrm{NADP}{ }^{+}$, $5.7 \mathrm{mM} \mathrm{D}$-Hiv, and enzyme in a final volume of $3.0 \mathrm{~mL}$. The increase in the rate of reduction of NADP ${ }^{+}$ due to oxidation of D-Hiv was measured at $340 \mathrm{~nm}$ $\left(45^{\circ} \mathrm{C}\right)[18,19,29]$.

\section{Characterization of recombinant KivRFp and biochemical properties}

The purified KivRFp was subjected to a series of biochemical analysis, including determine the $\mathrm{pH}$ optimum, temperature optimum, and effects of various detergents and metal ions. All measurements were carried out in triplicate. The values were the mean of the data. The substrate specificity of the purified KivRFp protein was performed using the following substrates: pyruvate, 2-Kiv, 2-ketovalerate, 2-ketobutyrate, 2-ketocapronate and 2-ketoglutarate.

The optimum temperature of purified KivRFp was determined by assaying reductase activities in a $50 \mathrm{mM}$ sodium phosphate buffer $(\mathrm{pH}$ 8.0) for a temperature range of $25-65^{\circ} \mathrm{C}$, in which $2-\mathrm{Kiv}(0.7 \mathrm{mM})$ acted as substrate. Optimal $\mathrm{pH}$ was determined by examining the activity of the enzyme after incubation at $40^{\circ} \mathrm{C}$ for 10 min using 2-Kiv $(0.7 \mathrm{mM})$ as substrate. The buffers used were: $50 \mathrm{mM}$ sodium phosphate buffer ( $\mathrm{pH}$ 5.0-8.0), $50 \mathrm{mM}$ Tris- $\mathrm{HCl}$ buffer ( $\mathrm{pH}$ 8.0-10.5).

Various metal ions $\left(\mathrm{CoCl}_{2}, \mathrm{CaCl}_{2}, \mathrm{ZnCl}_{2}, \mathrm{MgCl}_{2}\right.$, $\mathrm{K}_{2} \mathrm{SO}_{4}, \mathrm{FeSO}_{4}, \mathrm{CuCl}_{2}, \mathrm{MnCl}_{2}$, and $\mathrm{FeCl}_{3}$ ), and chelating agent EDTA at final concentrations of $5 \mathrm{mM}$ were added to the enzyme in $50 \mathrm{mM}$ sodium phosphate buffer $(\mathrm{pH}$ 8.0), that was assayed for 2-Kiv reductase activity following preincubation at $40^{\circ} \mathrm{C}$. Effect of detergents on reductase activity was determined by incubating the enzyme for $10 \mathrm{~min}$ at $40^{\circ} \mathrm{C}$ in $50 \mathrm{mM}$ sodium phosphate buffer ( $\mathrm{pH}$ 8.0), containing Tween-20, Tween-80, Triton X-100, $\beta$-mercaptoethanol, sodium dodecyl sulfate (SDS), cetyltrimethyl ammonium bromide (CTAB), diethylpyrocarbonate (DEPC), phenylmethanesulfonyl fluoride (PMSF), dimethyl sulfoxide (DMSO). The concentrations of metal ions, EDTA, detergents, and surfactants used were $5 \mathrm{mM}, 3 \mathrm{mM}$, and $0.5 \%(\mathrm{v} / \mathrm{v})$, respectively. The activity of the enzyme preparation in the absence of metal ions and detergents before incubation was defined as the $100 \%$ level.

\section{Nucleotide sequence accession number}

The DNA sequence of KivRFp from Fusarium proliferatum LF061 was deposited in GenBank under accession number of JQ922252. 


\section{Results}

Cloning and sequence analysis of KivRFp

We have recently cloned and functionally characterized beauvericin biosynthetic locus (bea) of the $F$. proliferatum LF061 genome (unpublished data). The FpBEAS gene encoding the beauvericin synthetase was found to be clustered with a gene (KivRFp) encoding a deduced putative 2-ketoisovalerate reductase (KivRFp). Amino acid sequence alignment indicated that KivRFp exhibited low identity with other ketopantoate reductases or hypothetical proteins in GenBank. KivRFp shared the highest sequence identity with the KIVR from $B$. bassiana ATCC 7159 (ACI30654, 61\% identity/75\% similarity), followed by the putative 2-ketopantoate reductase from Metarhizium acridum CQMa 102 (EFY89891, 56\% identity/70\% similarity). Similarity was also detected with the hypothetical or putative 2-dehydropantoate reductases from Gibberella zeae PH-1 (anamorph: F. graminearum) and F. oxysporum Fo5176 (EGU84839, 49\% identity; and EGU75687, 47\% identity, respectively), and also with functionally uncharacterized terminal reductase domain of the Xylaria sp. bassianolide synthetase (ABR28366, $30 \%$ identity $/ 48 \%$ similarity).

Various ketonate reductases contain the conserved GXGXXG nucleotide-binding signature. And also, a conserved glutamate (Glu), an asparagines (Asn) and a lysine (Lys), together constituting a catalytic triad [30,31]. The amino acid sequence alignment to other ketonate reductases retrieved from GenBank, identified the conserved motifs, including the putative GXGXXG nucleotidebinding signature and a Glu-Asn-Lys triad active site architecture (Figure 2). Thus, KivRFp probably uses a catalytic triad consisting of the glutamate (Glu405), the asparagine (Asn184) and the highly conserved lysine

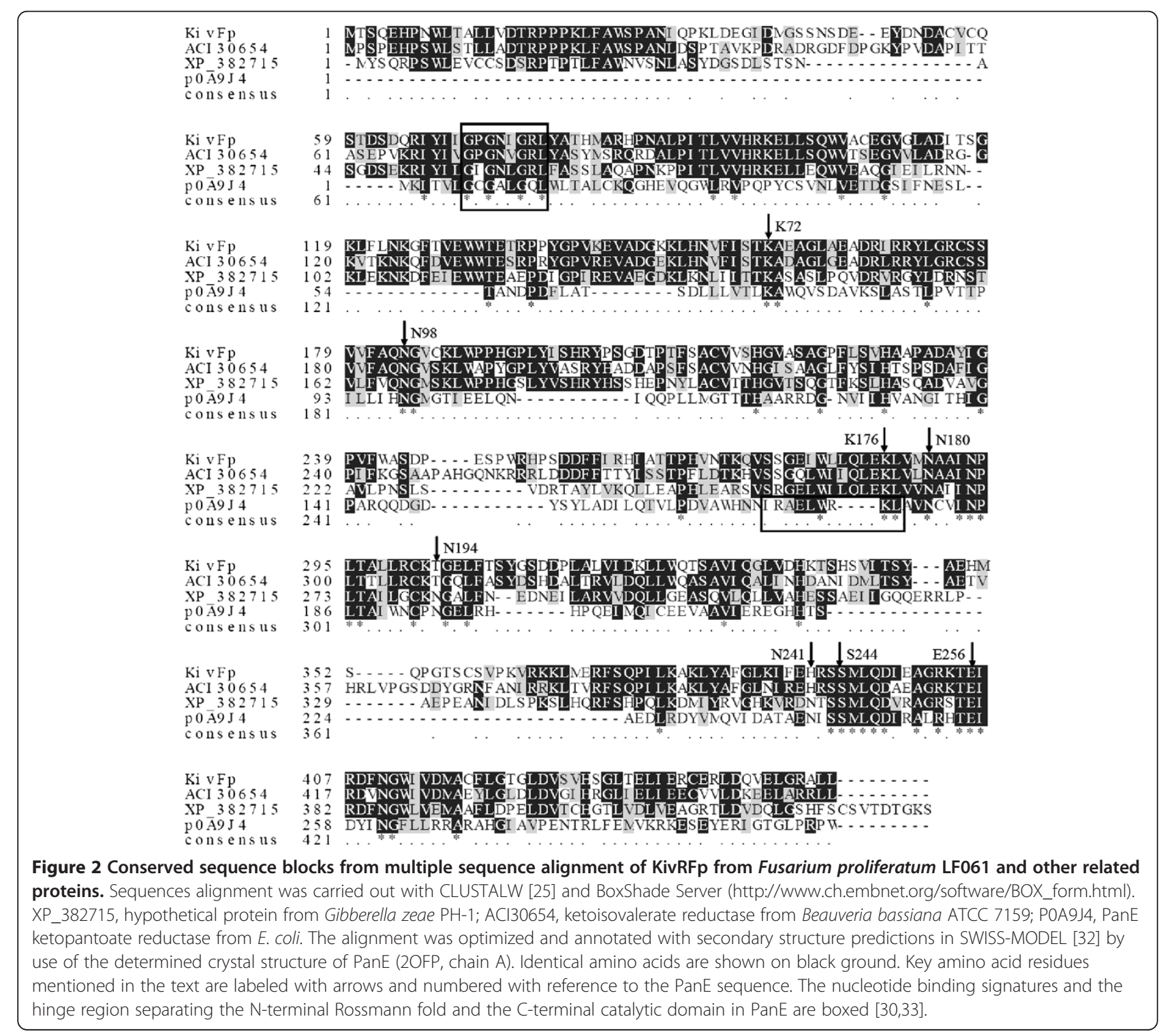


(Lys285) for catalysis. All these residues are conserved in the F. proliferatum KivRFp that might contribute to the formation of the active hole and involve directly in the catalytic process [30,31]. Furthermore, to clarify the phylogenetic relationship of the KivRFp with other ketonate reductases, a neighbor joining phylogenetic tree was constructed using the amino acid sequence of the ketonate reductive enzymes. As shown in Figure 3. In this tree, KivRFp formed a distinct group with the KIVR of B. bassiana ATCC7159 (ACI30654), which is located closest to the branch of putative 2-ketopantoate reductase of Metarhizium acridum CQMa 102 (EFY89891), hypothetical protein of Trichoderma virens Gv29-8 and Cordyceps militaris CM01 (accession number EHK17663 and EGX96948 respectively). Moreover, KivRFp showed sequence and the structural similarity with ketopantoate reductases [32,33], but not with D-lactose or D-hydroxyisocaproate dehydrogenase [20]. These results suggest that the KivRFp is a new member of family ketonate reductases.

\section{Expression and purification of recombinant KivRFp}

To investigate the property of this KivRFp, optimized KivRFp gene was expressed as an N-terminal His-tag fusion protein using pET-28a $(+)$ expression system in E. coli BL21(DE3). The recombinant protein was analyzed by SDS-PAGE and Coomassie brilliant blue staining (Figure 4). These results indicate that recombinant KivRFp protein is expressed ( $\mathrm{Mw}$, about $52 \mathrm{kDa}$ ), as which correlated well to the predicted full length of KivRFp. The purity of the purified protein was more than $98 \%$ according to SDS-PAGE analysis.

\section{Substrate and cofactor specificity of KivRFp}

We expressed KivRFp as a hexahistidine-tagged (Histagged) protein and investigated its substrate specificity. The enzyme exhibited the highest activity towards 2-Kiv as substrate, while the homologues compounds 2-ketovalerate, pyruvate, 2-ketocapronate, 2-ketoglutarate and

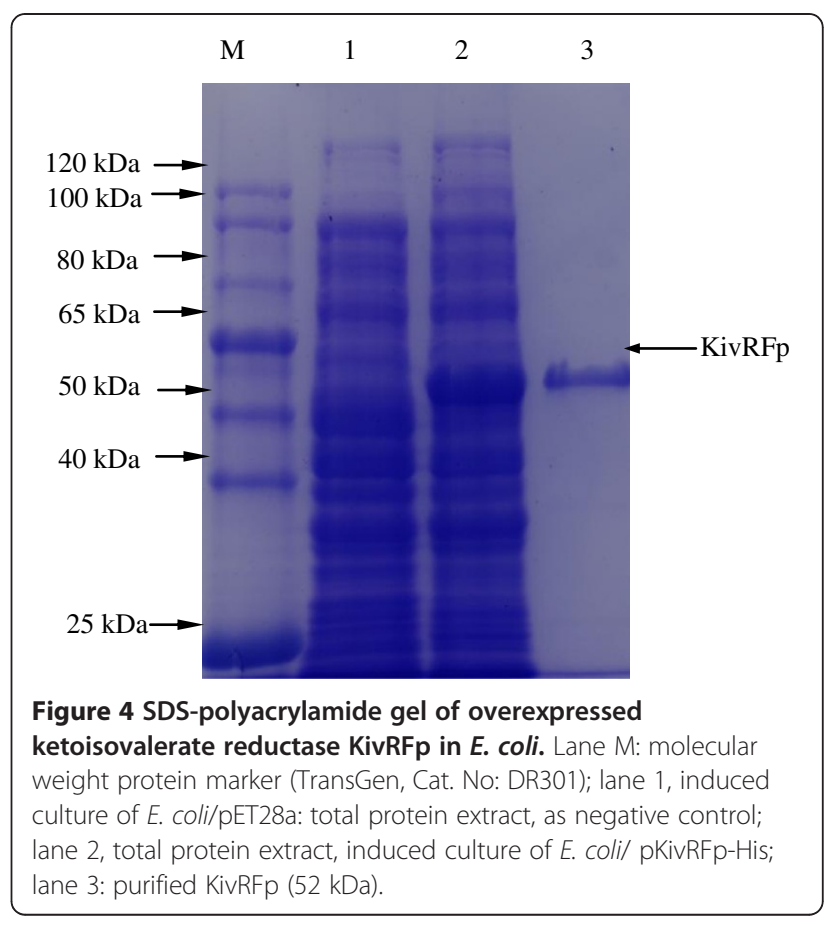

2-ketobutyrate were shown to be poor substrates (Figure 5A). And also, the KivRFp showed higher coenzyme specificity with respect to NADPH than $\mathrm{NADH}$ (Figure 5B), which is a little different from highly specific D-hydroxyisovalerate dehydrogenase from enniatin producer F. sambucinum.

\section{Effect of temperature and pH on KivRFp}

Reduction of 2-Kiv activity of KivRFp was determined from $25^{\circ} \mathrm{C}$ to $65^{\circ} \mathrm{C}$. The purified KivRFp showed highest activity at $35^{\circ} \mathrm{C}$. However, the activity of KivRFp reduced quickly above $55^{\circ} \mathrm{C}$ (Figure 6). Thermostability analysis showed that KivRFp was not stable and lost activity in less than $10 \mathrm{~min}$ at $60^{\circ} \mathrm{C}$ (data not shown). And also, the reductase showed activity in a rather broader $\mathrm{pH}$ range of 6.5-9.5. Maximal activity was observed at $\mathrm{pH} 7.5$ and

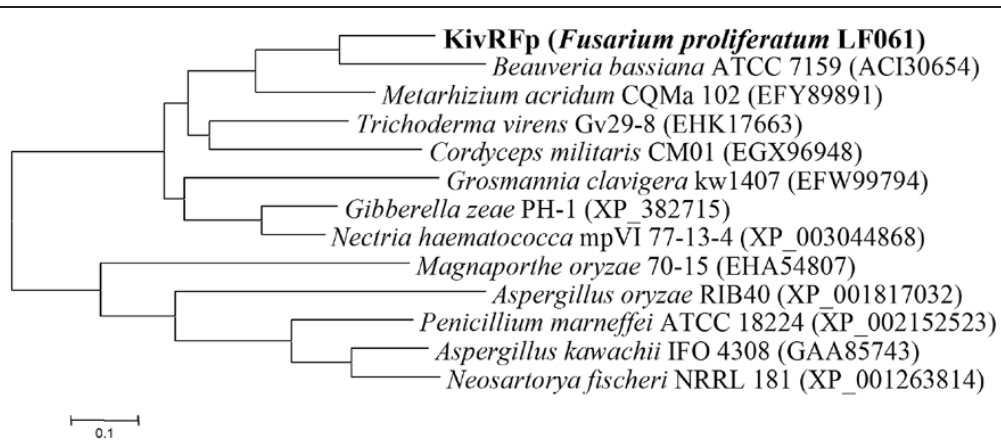

Figure 3 Phylogenetic analysis of KivRFp and closely related proteins. Phylogenetic analysis was performed using the program MEGA4.0 $[23,24]$. Except for KivRFp, the protein sequences for dehydrogenase were retrieved from GenBank (http://www.ncbi.nlm.nih.gov). The numbers at node indicate the bootstrap percentages of 1000 resamples. 

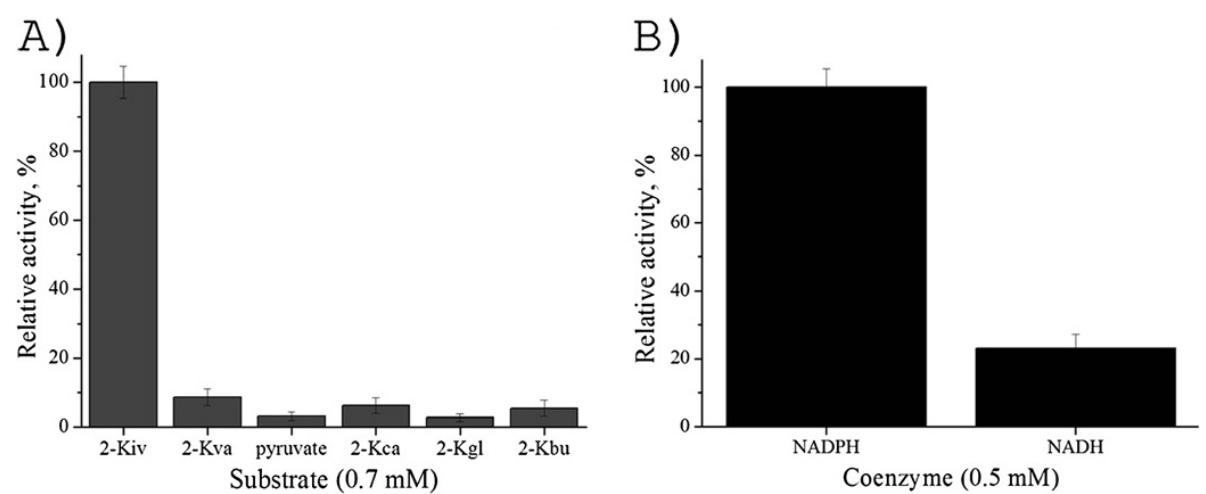

Figure 5 Substrate specificity of overexpressed and purified ketoisovalerate reductase KivRFp. 5 A). Specific activity of reductive reaction of different homologous compounds using NADPH (0.29 mM) as coenzyme. 2-ketoisovalerate (2-Kiv), 2-ketovalerate (2-Kva), pyruvate, 2-ketocapronate (2-Kca), 2-ketoglutarate (2-Kgl), and 2-ketobutyrate (2-Kbu). 5B). Specific activity of reductive reaction of different coenzymes using 2-Kiv $(0.7 \mathrm{mM})$ as substrate. Relative activity was shown as the percentage of the activity of the activity towards 2-ketoisovalerate. All measurements were performed in triplicate.

lost activity at $\mathrm{pH} 10.5$ using 2-ketoisovalerate and NADPH as substrates (Figure 7). However, the optimum temperature and $\mathrm{pH}$ of oxidative reaction were found to be $40^{\circ} \mathrm{C}$ and 8.5 respectively (not shown).

\section{Effect of metal ions on KivRFp}

The effects of metal ions and ethylenediamine tetraacetic acid (EDTA) on the KivRFp reductase activity were investigated by measuring the residual enzyme activity in their presence and depicted in Table 2. Among metal ions tested, the reductase activity was increased by $\mathrm{Mg}^{2+}$ (195\%), $\mathrm{Ca}^{2+}(169 \%)$ and a slight increase observed by $\mathrm{K}^{+}$
(123\%). Furthermore, the reduction activity was inhibited by $\mathrm{Fe}^{2+}$ and $\mathrm{Co}^{2+}$, moreover, almost totally inhibited by $\mathrm{Cu}^{2+}, \mathrm{Mn}^{2+}, \mathrm{Zn}^{2+}$ and $\mathrm{Fe}^{3+}$ ( from $3 \%$ to $9 \%$ residual activity respectively), while the chelating agent EDTA had little effect, suggesting $\mathrm{Mg}^{2+}$ and $\mathrm{Ca}^{2+}$ are not determining factors, though they could promote the reductase enzyme activity.

\section{Effect of detergents and enzyme inhibitors on KivRFp}

The effects of detergents and enzyme inhibitors on ketoisovalerate reductase (KIVR) reduction activity are shown in Table 3. A significant increase in reductive

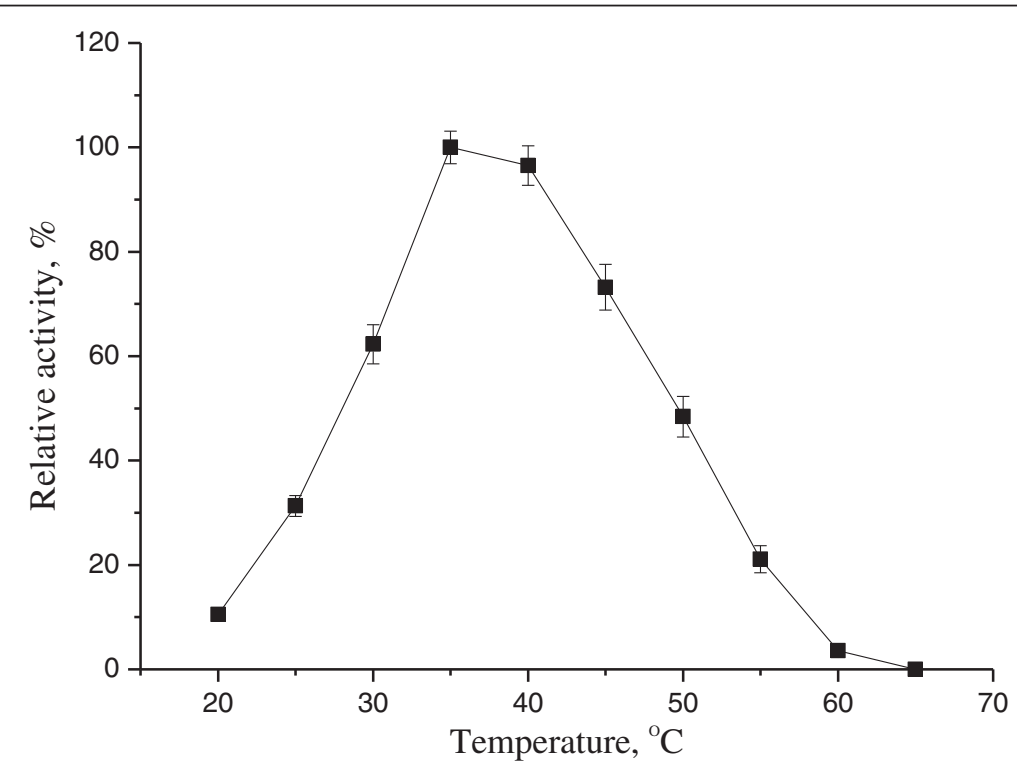

Figure 6 Apparent temperature optimum of ketoisovalerate reductase KivRFp. Relative activity of ketoisovalerate reduction at different temperatures by purified KivRFp. The activity was determined at different temperatures at pH 8.0 in 50 mM sodium phosphate buffer. The activity at $35^{\circ} \mathrm{C}$ was set as $100 \%$. All measurements were performed in triplicate. 


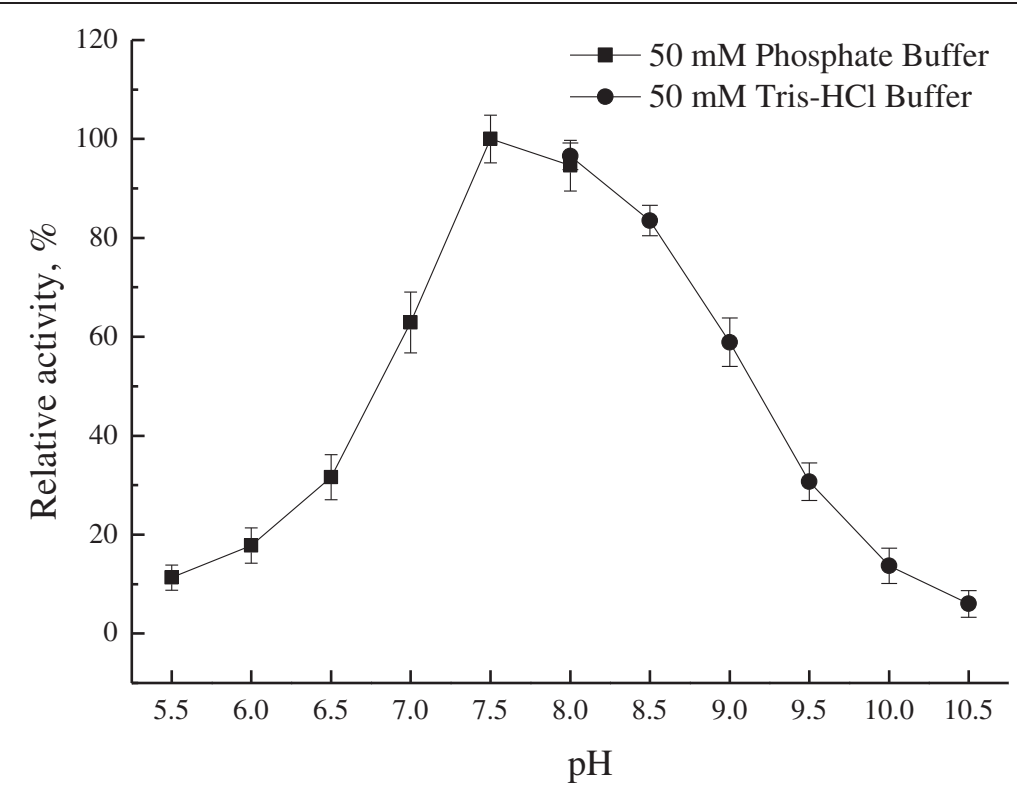

Figure 7 Effect of $\mathrm{pH}$ on the purified ketoisovalerate reductase KivRFp. Relative activity of ketoisovalerate reduction was performed in various $\mathrm{pH}$ buffers at $35^{\circ} \mathrm{C}$ (pH 5.0-8.0, $50 \mathrm{mM}$ sodium phosphate buffer; $\mathrm{pH}$ 8.0-10.5, $50 \mathrm{mM}$ Tris-HCl buffer). The activity at pH 7.5 was set as $100 \%$. All measurements were performed in triplicate.

activity was observed with addition of $3 \mathrm{mM}$ DTT (127\%), Tween-80 (176\%), DEPC (114\%), and Tween-20 (129\%), after $0.5 \mathrm{~h}$ preincubation with detergents at $37^{\circ} \mathrm{C}$, which might help the substrate interaction with the catalytic triad. Moreover, $0.5 \% \quad \beta$-mercaptoethanol did not affect the reductive activity (102\%), whereas CTAB, PMSF, Triton X-100, and SDS strongly inhibited enzymatic activity of KivRFp. The activity inhibition of KivRFp by PMSF suggests that serine is involved in the catalytic center, since PMSF could mimic the first transition state in ester bond hydrolysis presumably by linking to the hydroxyl group of serine in the active site covalently. The enzymatic activity of KivRFp is totally

Table 2 Effect of metal ions on esterase activity

\begin{tabular}{llc}
\hline Compounds & Concentration (mM) & Relative activity (\%) \\
\hline Control & 0 & $100.0 \pm 1.7$ \\
$\mathrm{CoCl}_{2}$ & 5 & $16.4 \pm 2.9$ \\
$\mathrm{~K}_{2} \mathrm{SO}_{4}$ & 5 & $123.2 \pm 3.4$ \\
$\mathrm{FeSO}_{4}$ & 5 & $4.9 \pm 1.6$ \\
$\mathrm{CuCl}_{2}$ & 5 & $7.8 \pm 2.7$ \\
$\mathrm{MnCl}_{2}$ & 5 & $6.2 \pm 1.3$ \\
$\mathrm{EDTA}$ & 5 & $87.7 \pm 3.2$ \\
$\mathrm{FeCl}_{3}$ & 5 & $7.9 \pm 3.4$ \\
$\mathrm{CaCl}_{2}$ & 5 & $169.1 \pm 3.7$ \\
$\mathrm{ZnCl}_{2}$ & 5 & $3.7 \pm 1.8$ \\
$\mathrm{MgCl}_{2}$ & 5 & $195.6 \pm 2.7$ \\
\hline $\mathrm{Atvity}$ & $50 u t$
\end{tabular}

Activity without metal ions was set as $100 \%$. All measurements were repeated three times. inhibited by ionic detergent and the probable reasons for these are ionic detergents such as SDS inhibits both inter- and intra-molecular protein-protein interaction.

\section{Discussion}

Ketoisovalerate reductases are types of oxidoreductases which are widely distributed from prokaryotes to eukaryotes and which are involved in primary and second metabolism [17,20,34-36]. 2-hydroxyisovalerate is a common 2-hydroxycarboxylate constituent of depsipeptides. Reduction of Kiv in fungi provides Hiv, a precursor for the biosynthesis of nonribosomal

Table 3 Effect of detergents and enzyme inhibitors on esterase activity

\begin{tabular}{lcr}
\hline Compounds & Concentration & Relative activity (\%) \\
\hline Control & 0 & $100.0 \pm 2.1$ \\
DTT & $3 \mathrm{mM}$ & $126.9 \pm 4.9$ \\
CTAB & $3 \mathrm{mM}$ & $42.7 \pm 2.2$ \\
DEPC & $3 \mathrm{mM}$ & $113.6 \pm 2.7$ \\
PMSF & $3 \mathrm{mM}$ & $53.6 \pm 4.1$ \\
SDS & $3 \mathrm{mM}$ & $6.3 \pm 1.9$ \\
$\beta$-mercaptoethanol & $0.5 \%$ & $101.7 \pm 2.6$ \\
Triton X-100 & $0.5 \%$ & $60.8 \pm 4.6$ \\
Tween-80 & $0.5 \%$ & $176.4 \pm 2.1$ \\
Tween-20 & $0.5 \%$ & $128.7 \pm 3.3$ \\
\hline
\end{tabular}

Activity without detergents and enzyme inhibitors was set as $100 \%$. All measurements were repeated three times. 
depsipeptides and aroma compounds. Fungal cyclooligomer depsipeptides synthetase A domains incorporate free 2-hydroxycarboxylate precursors for the synthesis of CODs, preformed by dissociated, independent, monofunctional, NADPH-dependent enzymes with high chiral specificity, exemplified by the D-Hiv dehydrogenase purified from the enniatin producer F. sambucinum (teleomorph: G. pulicaris) [17,19]. In contrast, known bacterial depsipeptide synthetases employ A domains that activate and load 2-ketocarboxylates as $\beta$ ketoacyl thioesters onto their cognate $\mathrm{T}$ domains, and utilize integrated ketoacyl reductase domains for the synthesis of 2-hydroxycarboxylate thioesters. This might suggest the biosynthesis of 2-hydroxycarboxylate precursors in bacteria and fungi may represent example of convergent evolution [20]. This enzyme, which plays a key role in beauvericin biosynthesis, catalyzes the reversible reaction of 2-Kiv to D-Hiv using $\mathrm{NAD}(\mathrm{P}) \mathrm{H}$ as a cofactor. 2-ketoisovalerate reductase differs from other NADPH-dependent oxidoreductases with broad substrate specificity by its high affinity for 2Kiv (Figure 5A). The high specificity of KivRFp may also explain the fact that D-Hiv is the exclusive hydroxy acid component in beauvericin isolated from Fusaria (Logrieco A et al., 1998). To our knowledge this is the first report about the heterologous production of a ketoisovalerate reductase with higher substrate specificity for 2-Kiv.

KivRFp, which was cloned, expressed, and purified, was subjected to molecular and biochemical experiments in this study. The F. proliferatum KivRFp exhibits high amino acid sequence similarity to $B$. bassiana KIVR and deduced dehydropantoate reductases of fungi, together with low but significant sequence similarity predicted secondary structure to prokaryotic ketopantoate reductases such as PanE of E. coli $[20,30,31,36]$. KivRFp and the related B. bassiana KIVR are predicted to share the $\mathrm{N}$-terminal $\alpha \beta$-Rossmann fold and the C-terminal $\alpha$-helical catalytic domain architecture of the E. coli ketopantoate reductase. In the amino acid sequence of KivRFp, the conserved catalytic triad (Glu-Asn-Lys) and substrates stabilization active amino acids of ketonate oxidoreductases are still the same. In ketopantoate reductase PanE from E. coli, the protonated Lys176 (KivRFp: Lys285, Figure 2) could function as a general acid to polarize the carbonyl in the direction of ketopantoate reduction, facilitates the hydride transfer from $\mathrm{NADPH}$ to the C2 carbonyl of ketopantoate, and protonates the intermediate alkoxide [20,31]. Asn98 (KivRFp: Asn184) forms hydrogen binding both with the carboxylate of the ketopantoate and with the ribose of the nicotinamide: this orients the cofactor. And also, Glu256 (KivRFp: Glu405) and Lys72 (KivRFp: Lys158) further participate substrate binding to the NADPH [20]. Further stabilization is provided by a hydrogen bond between the Ser244 (KivRFp: Ser393) and carboxyl group of the ketopantoate, and between Asn180 (KivRFp: Asn289) and the $a$-hydroxy group of the product. And also, KivRFp showed sequence and the structural similarity with ketopantoate reductases, but not with D-lactose or D-hydroxyisocaproate dehydrogenase [20]. Further biochemical assay demonstrated that Fusarium KivRFp could utilize NADPH and $\mathrm{NADH}$ as coenzyme, while the NADPH is preferred (Figure 5B). In accordance to our results, bacterial chiral enzymes belonging to the D-ketoacid reductases superfamily also could utilize $\mathrm{NADH}$ as coenzyme, and also feature a conserved Arg-(Asp/Glu)-His active site triad [20,37]. Based on the phylogenetic, molecular, and biochemical analysis results, we propose that KivRFp is a new member of family ketonate oxidoreductases. Future work will establish the structure of this enzyme to gain more information about its catalytic mechanism.

\section{Conclusion}

In conclusion, we identified a new ketoisovalerate reductase KivRFp from the depsipeptide-producing fungus $F$. proliferatum. KivRFp is expected to show high potential for downstream biotechnological applications including synthetic organic chemistry and combinatorial biosynthesis beauvericin homologues. This was confirmed by its extensive biochemical characterization, which revealed the enzymes substrate specificity, wide $\mathrm{pH}$ and temperature spectra, and also, stability towards addictives including metal ions and detergents. Enlargement of the ketoisovalerate reductase pool can be an immediate source of genetic modification, or yield enzymes that can be further specialized by directed evolution, and also, this would optimize their industrial applications.

\section{Abbreviation}

BEA: Beauvericin; CODs: Cyclooligomer depsipeptides; NRPS: Nonribosomal peptide synthetases; D-Hiv: D-hydroxyisovalerate; 2-Kiv: 2-ketoisovalerate; CODS: Cyclooligomer depsipeptides synthetase; D-HivDH: D-Hiv dehydrogenase; 2-KivR: 2-Kiv reductase.

\section{Competing interests}

The authors declare that they have no competing interests.

\section{Authors' contributions}

TZ participated in the design of experiments, and carried out the study and drafted the manuscript. XPJ, YZ, ML, HG, JTL and LXZ participated in its design and coordination and helped to draft the manuscript. All authors read and approved the final manuscript.

\section{Authors' information}

TZ, XPJ, YZ, ML, HG and LXZ, Chinese Academy of Sciences Key Laboratory of Pathogenic Microbiology and Immunology, Institute of Microbiology, Chinese Academy of Sciences, Beijing 100190, China. JTL, deceased.

\section{Acknowledgments}

The authors are very grateful to Elizabeth Ashforth for critical reading and helpful discussions. This work was supported in part by grants from National Natural Science Foundation of China $(31100075,81102362,31170095$, 
31000004), the CAS Pillar Program (XDA04074000) and the Ministry of Science and Technology of China (2011ZX11102-011-11, 2007DFB31620,). L. Z. is an Awardee for National Distinguished Young Scholar Program in China.

\section{Author details}

${ }^{1}$ Chinese Academy of Sciences Key Laboratory of Pathogenic Microbiology and Immunology, Institute of Microbiology, Chinese Academy of Sciences, Bei'er Tiao Road, Zhongguancun Haidian District, Beijing 100190, China. ${ }^{2}$ Graduate University of Chinese Academy of Sciences, Beijing 100190, China.

Received: 13 June 2012 Accepted: 15 August 2012

Published: 23 August 2012

\section{References}

1. Hamill RL, Higgens CE, Boaz HE, Gorman M: Structure of beauvericin, a new depsipeptide antibiotic toxic to Artemia salina. Tetrahedron Lett 1969, 49:4255-4258

2. Shemyakin MM, Ovchinnikov YA, Kiryushkin AA, Ivanov VT: Concerning the structure of enniatin-B. Tetrahedron Lett 1962, 7:301-305.

3. Pedras MS, Irina Zaharia L, Ward DE: The destruxins: synthesis, biosynthesis, biotransformation, and biological activity. Phytochemistry 2002, 59:579-596.

4. Kanaoka M, Isogai A, Murakoshi S, Ichinoe M, Suzuki A, Tamura S: Bassianolide, a new insecticidal cyclodepsipeptide from Beauveria bassiana and verticillium lecanii. Agric Biol Chem 1978, 42:629-635.

5. Levy D, Bluzat A, Seigneuret M, Rigaud JL: Alkali cation transport through liposomes by the antimicrobial fusafungine and its constitutive enniatins. Biochem Pharmacol 1995, 50:2105-2107.

6. Luangsa-Ard JJ, Berkaew P, Ridkaew R, Hywel-Jones NL, Isaka M: A beauvericin hot spot in the genus Isaria. Mycol Res 2009, 113:1389-1395.

7. Logrieco A, Moretti A, Castella G, Kostecki M, Golinski P, Ritieni A, Chelkowski J: Beauvericin production by Fusarium species. Appl Environ Microbiol 1998, 64:3084-3088

8. Molnar I, Gibson DM, Krasnoff SB: Secondary metabolites from entomopathogenic Hypocrealean fungi. Nat Prod Rep 2010, 27:1241-1275.

9. Sussmuth R, Muller J, von Dohren H, Molnar I: Fungal cyclooligome depsipeptides: from classical biochemistry to combinatorial biosynthesis. Nat Prod Rep 2011, 28:99-124.

10. Zhang LX, Yan KZ, Zhang Y, Huang R, Bian J, Zheng CS, Sun HX, Chen ZH, Sun $N$, An R, et al: High-throughput synergy screening identifies microbial metabolites as combination agents for the treatment of fungal infections. Proc Nat Acad Sci USA 2007, 104:4606-4611.

11. Fukuda T, Arai M, Tomoda H, Omura S: New beauvericins, potentiators of antifungal miconazole activity, produced by Beauveria sp FKI-1366 - II. Structure elucidation. J Antibiot 2004, 57:117-124.

12. Lee $H S$, Song HH, Ahn JH, Shin CG, Lee GP, Lee C: Statistical optimization of growth medium for the production of the entomopathogenic and phytotoxic cyclic depsipeptide beauvericin from Fusarium oxysporum KFCC11363P. J Microbiol Biotech 2008, 18:138-144.

13. Crowell PL, Miller RH, Harper AE: Measurement of plasma and tissue-levels of branched-chain alpha-keto acids by Gas-liquid chromatography. Method Enzymol 1988, 166:39-46.

14. Kato M, Wynn RM, Chuang JL, Brautigam CA, Custorio M, Chuang DT: A synchronized substrate-gating mechanism revealed by cubic-core structure of the bovine branched-chain alpha-ketoacid dehydrogenase complex. EMBO J 2006, 25:5983-5994.

15. Gasking AL, Edwards WTE, Hobsonfrohock A, Elia M, Livesey G: Quantitative high-performance liquid-chromatographic analysis of branched-chain 2-keto acids in biological samples. Method Enzymol 1988, 166:20-27.

16. Duggleby RG, Pang SS: Acetohydroxyacid synthase. J Biochem Mol Biol 2000, 33:1-36.

17. Lee C, Gorisch H, Kleinkauf H, Zocher R: A highly specific Dhydroxyisovalerate dehydrogenase from the enniatin producer Fusarium sambucinum. I Biol Chem 1992, 267:11741-11744.

18. Zocher R: Purification of D-hydroxyisovalerate dehydrogenase from Fusarium sambucinum. Branched-Chain Amino Acids, Pt B 2000, 324:293-301.

19. Lee C, Zocher R: The biochemical characterization of Dhydroxyisovalerate dehydrogenase, a key enzyme in the biosynthesis of enniatins. J Biochem Mol Biol 1996, 29:493-499.
20. $\mathrm{XU}$ YQ, Wijeratne EMK, Espinosa-Artiles P, Gunatilaka AAL, Molnar I: Combinatorial mutasynthesis of scrambled beauvericins, cyclooligomer depsipeptide cell migration inhibitors from Beauveria bassiana. Chem Bio Chem 2009, 10:345-354

21. Sambrook J: DW R: Molecular Cloning: A Laboratory Manual. 3rd edition. New York: Cold Spring Harbor Laboratory Press; 2001.

22. Birnboim HC, Doly J: A rapid alkaline extraction procedure for screening recombinant plasmid DNA. Nucleic Acids Res 1979, 7:1513-1523.

23. Saitou N, Nei M: The neighbor-joining method - a new method for reconstructing phylogenetic trees. Mol Biol Evol 1987, 4:406-425.

24. Tamura K, Dudley J, Nei M, Kumar S: MEGA4: Molecular Evolutionary Genetics Analysis (MEGA) software version 4.0. Mol Biol Evol 2007, 24:1596-1599.

25. Thompson JD, Gibson TJ, Plewniak F, Jeanmougin F, Higgins DG: The CLUSTAL_X windows interface: flexible strategies for multiple sequence alignment aided by quality analysis tools. Nucleic Acids Res 1997, 25:4876-4882

26. Grote A, Hiller K, Scheer M, Munch R, Nortemann B, Hempel DC, Jahn D: JCat: a novel tool to adapt codon usage of a target gene to its potential expression host. Nucleic Acids Res 2005, 33:W526-W531.

27. Puigbo P, Guzman E, Romeu A, Garcia-Vallve S: OPTIMIZER: a web server for optimizing the codon usage of DNA sequences. Nucleic Acids Res 2007, 35:W126-W131.

28. Brown RE, Jarvis KL, Hyland KJ: Protein measurement using bicinchoninic acid - elimination of interfering substances. Anal Biochem 1989, 180:136-139.

29. Lee C, Goerisch H, Zocher R: The kinetic investigation of Dhydroxyisovalerate dehydrogenase from Fusarium sambucinum. J Biochem Mol Biol 2000, 33:228-233.

30. Ciulli A, Chirgadze DY, Smith AG, Blundell TL, Abell C: Crystal structure of Escherichia coli ketopantoate reductase in a ternary complex with NADP $(+)$ and pantoate bound - Substrate recognition, conformational change, and cooperativity. J Biol Chem 2007, 282:8487-8497.

31. Zheng RJ, Blanchard JS: Identification of active site residues in E. coli ketopantoate reductase by mutagenesis and chemical rescue. Biochemistry 2000, 39:16244-16251.

32. Arnold K, Bordoli L, Kopp J, Schwede T: The SWISS-MODEL workspace: a web-based environment for protein structure homology modelling Bioinformatics 2006, 22:195-201.

33. Lobley CMC, Ciulli A, Whitney HM, Williams G, Smith AG, Abell C, Blundell $T L:$ The crystal structure of Escherichia coli ketopantoate reductase with NADP(+) bound. Biochemistry 2005, 44:8930-8939.

34. Xu Y, Orozco R, Wijeratne EM, Gunatilaka AA, Stock SP, Molnar I: Biosynthesis of the cyclooligomer depsipeptide beauvericin, a virulence factor of the entomopathogenic fungus Beauveria bassiana. Chem Biol 2008, 15:898-907.

35. Peters J, Zelinski T, Kula MR: Studies on the distribution and regulation of microbial keto ester reductases. Appl Microbiol Biotechnol 1992, 38:334-340

36. Heider J, Mai XH, Adams MWW: Characterization of 2-ketoisovalerate ferredoxin oxidoreductase, a new and reversible coenzyme A-dependent enzyme involved in peptide fermentation by hyperthermophilic archaea. J Bacteriol 1996, 178:780-787.

37. Kletzin A, Adams MWW: Molecular and phylogenetic characterization of pyruvate and 2-ketoisovalerate ferredoxin oxidoreductases from Pyrococcus furiosus and pyruvate ferredoxin oxidoreductase from Thermotoga maritima. J Bacteriol 1996, 178:248-257.

doi:10.1186/1472-6750-12-55

Cite this article as: Zhang et al: Cloning and characterization of a novel 2-ketoisovalerate reductase from the beauvericin producer Fusarium proliferatum LF061. BMC Biotechnology 2012 12:55. 